\title{
Artificial potential field based anti-saturation positioning obstacle avoidance control for wheeled robots
}

pengfei zhang ( $\square$ peng-fei_zhang@zjhu.edu.cn )

Huzhou University https://orcid.org/0000-0003-1628-4450

\section{Tingting Yang}

Huzhou University

\section{Yichao Ma}

Huzhou University

\section{Research Article}

Keywords: Wheeled robots , Anti-saturation positioning control , Obstacle avoidance control , Artificial potential field method, Barrier Lyapunov Function

Posted Date: February 8th, 2022

DOI: https://doi.org/10.21203/rs.3.rs-1298374/v1

License: (a) (1) This work is licensed under a Creative Commons Attribution 4.0 International License. Read Full License 


\title{
Artificial potential field based anti-saturation positioning obstacle avoidance control for wheeled robots
}

\author{
Tingting Yang · Yichao Ma • Pengfei Zhang
}

Received: date / Accepted: date

\begin{abstract}
This paper studies the anti-saturation positioning and obstacle avoidance control of wheeled robots. First, by modifying the existing control law based on the kinematics model, a continuous function is proposed to overcome the velocity saturation problem, then the positioning control law ensuring the continuity and avoiding chattering phenomenon caused by switching control is proposed. Afterwards, a Gaussian function and a switching function are introduced to guarantee the smoothness of the artificial potential field function, and the positioning obstacle avoidance control law based on Barrier Lyapunov Function is constructed to reach the target point more stably. Finally, the effectiveness and practicability of the control algorithm are verified by simulations.
\end{abstract}

Keywords Wheeled robots · Anti-saturation positioning control · Obstacle avoidance control · Artificial potential field method · Barrier Lyapunov Function

\section{Introduction}

In recent years, robots have played an increasingly important role in daily life. The research on the application of robots in logistics, education, medical and other fields is also more favored by the majority of scholars [1]. For example, some hospitals use robots to replace manual product delivery and other tasks, and hotels use self-service robots to deliver dishes to guests [2]. Rescuers use robots for search tasks in harsh environments [3].

\section{P.F. Zhang}

School of Engineering, Huzhou University, Huzhou 313000, China

E-mail: pengfei_zhang@zjhu.edu.cn
All of the above applications need to control the position of the robot by controlling the speed and angular velocity $(V, \omega)$ of the robot [4] [5]. This control action is also called point stability and has been extensively studied in the past few years. However, the robot will inevitably encounter obstacles during the movement. At this time, the obstacle avoidance problem should be added on the basis of the control law for robots' positioning [6] [7].

Siegwart [8] conducted a detailed analysis of the positioning problem and proposed a solution that considers the final direction of the robot. Still, the trajectory of the robot to the target point may not be optimal at this time. Malu [9] proposed a control law based on the robot kinematics model, which provides a reference speed for the PID control of the DC motor. Pourboghrat [10] proposed an adaptive control law for mobile robots. Using the method of tracking a reference trajectory, the error between the tracking trajectory and the actual trajectory gradually converges to zero, reaching a point of stability. C. de wit [5] proposed a model-based predictive control scheme, which can deal with state or input constraints. But Pourboghrat [10] and $\mathrm{C}$. de wit [5] did not consider the saturation of linear velocity and angular velocity.

The Khatib [12] virtual force method is widely used for robots to avoid obstacles because of its less calculation, good real-time performance and easy low-level control characteristics [13]. This method is called the artificial potential field method. $\mathrm{Pu}$ [14] added the distance between the robot and the target to the repulsive force field of the synthetic potential field method, which improved the local minimum problem of the artificial potential field method. Koren [15] based on the possible field method and its limitations, proposed a robot position control based on an obstacle avoidance 
algorithm. Still, the method used in this document has two ends. One is that the linear velocity is the maximum when the robot is away from the target point. This means that the robot can not turn as fast as possible when approaching obstacles, and it may encounter obstacles; Second, when the Angle error is substantial, the angular velocity will become an unacceptable value according to the control law.

Motivated by the aforementioned facts, the main contributions of this paper can be described as follows. Firstly, inspired by Villela [16] and Fabregas [17], an improved linear velocity algorithm overcoming the saturation problem based on [18] is introduced, and a positioning control law with the continuous linear velocity based on [17] is proposed. Compared with the literature [17], the controller designed in this paper is continuous, which avoids the chattering problem caused by the switching control method. Secondly, based on literature [19], a smooth switching function and Gaussian function are introduced to design the continuous artificial potential field function, and a Gaussian Barrier Lyapunov Function based on the artificial potential field method is constructed. In addition, combined with the proposed continuous linear velocity and smooth Gaussian Barrier Lyapunov Function, a novel positioning control method for wheel robots with obstacle avoidance is designed.

The paper is organized as follows. Section II gives the basic model and basic formula of positioning control and obstacle avoidance control. Section III designs the motion algorithm of positioning control and obstacle avoidance control and verifies the stability. Section IV conducts experimental simulations of the localization algorithm and the obstacle avoidance algorithm to verify the feasibility and superiority of the algorithms in this paper. Section V summarizes the research content of this paper and gives the future research directions.

\section{Problem formulation}

\subsection{Kinematic model}

The differential wheel mobile robot can control the direction of movement by changing the relative speed between the driving wheels. Define the left and right driving wheel speeds as $u_{L}$ and $u_{R}$. Assuming that the wheels roll without slipping, the kinematics model of the robot can be described as follows in the Cartesian coordinate system [20]:

$$
\begin{aligned}
& \dot{x}=u \cos \psi, \\
& \dot{y}=u \sin \psi, \\
& \dot{\psi}=\omega,
\end{aligned}
$$

Among them, $x, y \in R$ represents the position of the robot in the Cartesian coordinate system and $\psi$ represents the robot's heading angle, and its direction is perpendicular to the turning radius. $u$ represents the instantaneous linear velocity of the robot, and $u=$ $\frac{u_{L}+u_{R}}{2}, \omega$ represents the angular velocity of the robot, and $\omega=\frac{\left(u_{L}-u_{R}\right)}{l}$. Among them, $l$ is the distance between the two driving wheels.

\subsection{Control objectives}

\subsection{1 positioning control}

The wheel robot owns a limited velocity and a minimum turning radius. Assuming that its maximum linear velocity is $u_{\max }$ and its minimum turning radius is $R_{\min }$, in this case, the maximum angular velocity of the robot is $\omega_{\max }=\frac{u_{\max }}{R_{\min }}$. As shown in Fig. 1, for the purpose of

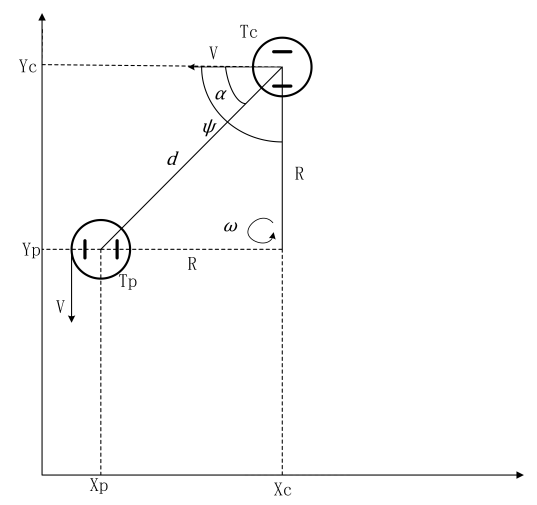

Fig. 1 Variables involved in positioning control problems.

positioning control, the distance $d$ and angle $\alpha$ between the points $T_{c}\left(x_{c}, y_{c}\right)$ and $T_{p}\left(x_{p}, y_{p}\right)$ are as follows

$$
\begin{aligned}
& d=\sqrt{\left(y_{p}-y_{c}\right)^{2}+\left(x_{p}-x_{c}\right)^{2}}, \\
& \alpha=\operatorname{atan} 2\left(y_{p}-y_{c}, x_{p}-x_{c}\right),
\end{aligned}
$$

Equations (2)-(3) mean that $y_{p}-y_{c}=d \sin (\alpha)$ and $x_{p}-x_{c}=d \cos (\alpha)$. Then define the angle error variable as follows

$e_{\psi}=\alpha-\psi$,

It can be seen that $e_{\psi} \in[-\pi, \pi]$. Taking the time derivative of equation (2) and combining it with equation (1), we get

$$
\begin{aligned}
\dot{d} & =-\frac{\dot{y}_{c}\left(y_{p}-y_{c}\right)+\dot{x}\left(x_{p}-x_{c}\right)}{d} \\
& =-u(\sin (\alpha) \sin (\psi)+\cos (\alpha) \cos (\psi)) \\
& =-u \cos (\alpha-\psi) .
\end{aligned}
$$


From (4), it has

$$
\begin{aligned}
\dot{e}_{\psi} & =\dot{\alpha}-\dot{\psi}=\frac{\left(y_{p}-y_{c}\right) \dot{x}_{c}-\left(x_{p}-x_{c}\right) \dot{y}_{c}}{d^{2}}-\omega \\
& =\frac{u \sin (\alpha-\psi)}{d}-\omega=\frac{u \sin \left(e_{\psi}\right)}{d}-\omega,
\end{aligned}
$$

Then we can combine the above two equations to obtain the following system

$\dot{d}=-u \cos \left(e_{\psi}\right)$,

$\dot{e}_{\psi}=\frac{u}{d} \sin \left(e_{\psi}\right)-\omega$.

Therefore, the robot positioning control problem is transformed into the stability control problem of state $d$ in (5). That is to say, by designing the linear velocity $u$ and angular velocity $\omega$ to realize $d \rightarrow 0$, then the robot can reach the target point and remain stable.

\subsubsection{Obstacle avoidance control}

The artificial potential field method mainly includes the gravitational potential field, repulsive potential field and allosteric potential field. As shown in Fig. 2, the target point generates the gravitational potential field $\phi_{f}(q)$, which produces the gravitational force, and its direction points from the robot to the target point. The obstacle produces the repulsive force field $\phi_{r}(q)$, which generates the repulsive force whose direction points from the obstacle to the robot. The combined force of gravitational and repulsive forces determines the direction of motion of the robot [21].

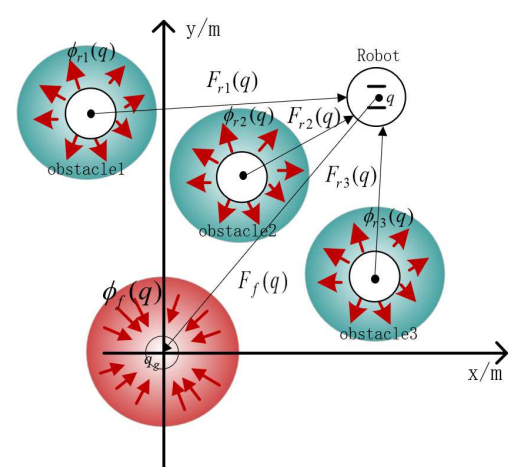

Fig. 2 Force analysis of artificial potential field method.

The gravitational field $\phi_{f}(q)$ and the attractive force $F_{f}(q)$ can be expressed as:

$\phi_{f}(q)=\frac{1}{2} \mu\left\|q-q_{g}\right\|^{2}$,

$F_{f}(q)=-\dot{\phi}_{f}(q)=-\mu\left\|q-q_{g}\right\|$ where $\mu$ is the gravitational field gain coefficient, $q$ is the current position of the robot, and $q_{g}$ is the position of the target point.

The repulsive force field $\phi_{r}(q)$ and the repulsive force $F_{r}(q)$ can be expressed as:

$$
\begin{aligned}
& \phi_{r}(q)=\left\{\begin{array}{cc}
\frac{1}{2} \beta\left(\frac{1}{\chi}-\frac{1}{\delta_{0}}\right)^{2}, & \chi \leq \delta_{0}, \\
0, & \chi>\delta_{0},
\end{array}\right. \\
& F_{r}(q)=-\dot{\phi}_{r}(q)=\left\{\begin{array}{cc}
\beta \frac{1}{2\left(\chi-\delta_{0}\right) \chi^{2}} \frac{\partial \chi}{\partial q}, & \chi \leq \delta_{0}, \\
0, & \chi>\delta_{0},
\end{array}\right.
\end{aligned}
$$

where $\beta$ is the gain coefficient of the repulsive force field, $\chi$ is the distance between the robot and the obstacle, and $\delta_{0}$ is the influence range of the obstacle repulsive force field, that is, the safety distance between the robot and the obstacle.

Therefore, the resultant force field $U(q)$ and the resultant force $F(q)$ received by the robot q are

$$
\begin{aligned}
& U(q)=\phi_{f}(q)+\phi_{r}(q), \\
& F(q)=F_{f}(q)+F_{r}(q) .
\end{aligned}
$$

Then, based on equation (8), the next objective is to achieve the positioning obstacle avoidance control for the wheeled robot, that is to say, the robot can reach the target point without obstacle collision.

\section{Main Results}

\subsection{Anti-saturation positioning controller design}

In [16], a linear velocity controller for the robot is designed based on the distance $d$ between the robot and the target point $T_{p}\left(x_{p}, y_{p}\right)$. The linear velocity designed in this literature reaches its saturation maximum at the beginning, and it only starts to decrease when $d$ is within a certain distance. Therefore, when the robot's heading angle is far from the target point, the robot will move forward with maximum speed, which will cause the malfunction of the controller and not reach the positioning control target. Therefore, when $d$ is large (large positioning error), we consider that the robot should reach the correct heading before the linear velocity is maximized. The literature [17] designed a deviation angle-based linear velocity controller, aiming to propose a velocity control guided by the correct heading angle to solve this problem.

Inspired by the literature [15] [17], we propose the following continuous controller,

$$
\begin{aligned}
& u=\tanh \left[K_{1} d p\left(e_{\psi}\right)\right] u_{\max }, \\
& \omega(t)=K_{p} \sin \left(e_{\psi}(t)\right)+K_{i} \int_{0}^{t} e_{\psi}(s) d s
\end{aligned}
$$


where

$p(x)=\frac{\pi^{2}-x^{2}}{\pi^{2}}$.

The parameters $K_{1}, K_{p}$ and $K_{i}>0$ meet the following constraints,

$0<u_{\max } K_{1}<K_{p}$,

$K_{p}+\sqrt{K_{i}} \pi<\omega_{\max }$,

It can be seen from (10) that $0 \leq p(x) \leq 1$ when $x \in$ $[-\pi, \pi]$.

\subsubsection{Stability analysis}

In order to facilitate subsequent controller design and analysis, the following related lemmas are introduced:

Lemma 1 (Barbalat theorem) Let $x:[0, \infty) \rightarrow R$ be continuouly first-order derivable and have limit when $t \rightarrow \infty$, if $\dot{x}$ is consistently continuous on $t \in[0, \infty)$, then $\lim _{t \rightarrow \infty} \dot{x}(t)=0$.

Lemma 2 Consider the system $\dot{x}(t)=f(t, x)$, assuming that $f(t, x)$ is segmentally continuous in $t$ and locally Lipschitz in $x$. Furthermore, assume that $f(t, 0)$ is consistently bounded for all $t \geq 0$. Definition $L$ : $\mathbb{R}^{n} \rightarrow \mathbb{R}$ is a continuous positive definite differentiable function such that $\dot{L}(x(t)) \leq-W(x), \forall t \geq 0, \forall x \in \mathbb{R}^{n}$, where $W(x)$ is a continuous positive semidefinite function, then $t \rightarrow \infty, W(x(t)) \rightarrow 0$.

Introducing the auxiliary state $z=\int_{0}^{t} e_{\psi}(s) d s$, the dynamic system (5) can be re-described as

$\dot{d}=-u \cos \left(e_{\psi}\right)$

$\dot{e}_{\psi}=\frac{u}{d} \sin \left(e_{\psi}\right)-\omega$,

$\dot{z}=e_{\psi}$,

Theorem 1 Considering the wheeled robot dynamic system (1) and designing the control law (9) based on the constraints (11), the dynamic system (12) is globally asymptotically stable, that is to say, $\lim _{t \rightarrow \infty} y_{p}-y=0$, $\lim _{t \rightarrow \infty} x_{p}-x=0$.

Proof. Substituting the controller (9) into system (12), we get

$$
\begin{gathered}
\dot{d}=- \\
\dot{e}_{\psi}=\frac{u_{\max } \tanh \left[K_{1} d p\left(e_{\psi}\right)\right] \cos \left(e_{\psi}\right),}{d}\left[K_{1} d p\left(e_{\psi}\right)\right] \\
-K_{p} \sin \left(e_{\psi}(t)\right)-K_{i} z
\end{gathered}
$$

$\dot{z}=e_{\psi}$
To analyze the stability, we first consider the subsystems (14)-(15) where states are $e_{\psi}$ and $z$, and the control input is $d(t)$. Design the following Lyapunov function,

$\mathcal{L}_{1}=\frac{e_{\psi}^{2}}{2}+\frac{K_{i} z^{2}}{2}$

Its time derivative is

$\dot{\mathcal{L}}_{1}=e_{\psi}\left\{\frac{u_{\max } \tanh \left[K_{1} d p\left(e_{\psi}\right)\right]}{d}-K_{p}\right\} \sin \left(e_{\psi}\right)$,

with $d \geq 0, K_{1}>0$. Because $-\pi<e_{\psi} \leq \pi, 0 \leq$ $p\left(e_{\psi}\right) \leq 1$, then $K_{1} d p\left(e_{\psi}\right) \geq 0$. Therefore

$$
\begin{aligned}
\dot{\mathcal{L}}_{1} & =e_{\psi}\left\{\frac{u_{\max } \tanh \left[K_{1} d p\left(e_{\psi}\right)\right]}{d}-K_{p}\right\} \sin \left(e_{\psi}\right) \\
& \leq e_{\psi}\left[u_{\max } K_{1} p\left(e_{\psi}\right)-K_{p}\right] \sin \left(e_{\psi}\right) \\
& =-W\left(e_{\psi}\right) .
\end{aligned}
$$

Then consider the subsystem (13) with state $d(t)$ and input $e_{\psi}$, and the following Lyapunov function is proposed,

$\mathcal{L}_{2}=\frac{d^{2}}{2}$

Its time derivative is

$$
\begin{aligned}
\dot{\mathcal{L}}_{2} & =-d u_{\max } \tanh \left[K_{1} d p\left(e_{\psi}\right)\right] \cos \left(e_{\psi}\right) \\
& \leq-\frac{\sqrt{2} d}{2} u_{\max } \tanh \left[K_{1} d p\left(e_{\psi}\right)\right] .
\end{aligned}
$$

Therefore, $\dot{\mathcal{L}}_{2} \leq 0$ and $\mathcal{L}_{2}=\frac{d^{2}}{2} \geq 0$. According to Lemma 1 , it has $d \rightarrow 0$ as $t \rightarrow \infty$. Therefore, the subsystem (13) is globally asymptotically stable. The proof is completed.

Remark 1 When $0 \leq u_{\max } \leq 1$, the stability proof of (16) is similar with literature [20], and the proof of saturation problem for state $\omega$ is consistent with literature [20], but the constraint condition of the stability proof of this paper is $u_{\max } K_{1} p\left(e_{\psi}\right)-K_{p} \leq 0$. Therefore, when $u_{\max }>1$, select appropriate $K_{1}$ and $K_{p}$, and the constraint conditions can also be established. The stability proof process of (17) is similar to that of literature [17].

Remark 2 In this paper, the control law is optimized on the robot linear velocity control law of literature [17]. The original control law requires switching between two velocity functions, but the controller designed in this paper is a continuous controller which avoids the chattering problem caused by the switching controller. The function $p\left(e_{\psi}\right)$ with the angular error $e_{\psi}$ as input is also improved to solve the robot reverse movement problem caused by the saturation of $e_{\psi}$. The better smoothness of $p\left(e_{\psi}\right)$ also makes the robot linear velocity change more smoothly. 
3.2 Anti-saturation obstacle avoidance controller design based on artificial potential field

\subsubsection{Improved artificial potential field function}

As shown in equation (7), the conventional repulsive field function needs to switch when $\chi=\delta_{0}$. When $\chi=\delta_{0}$, there are singularities in the repulsive function, which will cause the robot to wobble when entering or getting rid of the obstacles in the repulsive field. So in this paper a Gaussian function [22] is introduced to design the repulsive field function,

$g_{r}(q)=\left\{\begin{array}{c}\beta e^{-\frac{\chi^{2}}{\delta_{0}}}, \chi \leq \delta_{0}, \\ 0, \quad \chi>\delta_{0} .\end{array}\right.$

In order to solve the problem of unsmooth phenomenon caused by switching control, this paper introduces the n-th order smooth switching function $h(\chi)$ [23]:

$h(\chi)=\left\{\begin{array}{cc}1, & |\chi| \leq \chi_{b}, \\ \cos ^{n}\left[\frac{\pi}{2} \sin ^{n}\left(\frac{\pi}{2} \frac{\chi^{2}-\chi_{b}^{2}}{\chi_{a}^{2}-\chi_{b}^{2}}\right)\right], & \text { overwise } \\ 0, & |\chi| \geq \chi_{a},\end{array}\right.$

where $\chi_{a}$ is the maximum range of the repulsion field, $\chi_{b}$ is the minimum safety range of the obstacle under the influence of the repulsion field, and $\chi_{b}$ is greater than the radius of the circular obstacle.

Combining the above two equations, this paper constructs a new artificial potential field repulsion field function below,

$\phi_{f}(q)=\beta e^{-\frac{\chi^{2}}{\delta_{0}}} h(\chi)$,

and when multiple obstacles are present, it can be expressed as

$\sum_{i=1}^{N} \phi_{f}(q)=\sum_{i=1}^{N} \beta_{i} e^{-\frac{\chi_{i}^{2}}{\delta_{i}}} h\left(\chi_{i}\right)$.

The effect of $\beta_{i}, \delta_{i}$ parameter selection on the obstacle repulsion field is shown in Figure 3.

\subsubsection{Switching function analysis}

The switching function $h\left(\chi_{i}\right)$ as shown in Fig. 4 gradually starts working as $\left|\chi_{i}\right|$ decreases. As a result, the obstacle repulsive field gradually increases from $\chi_{a}$ until the repulsive field reaches its maximum at $\chi_{b}$. This represents the obstacle enters repulsive field $\chi_{a}$, the robot has a safe distance to avoid the the obstacle. Due to the smoothness of the switching function, the robot does not shake violently.

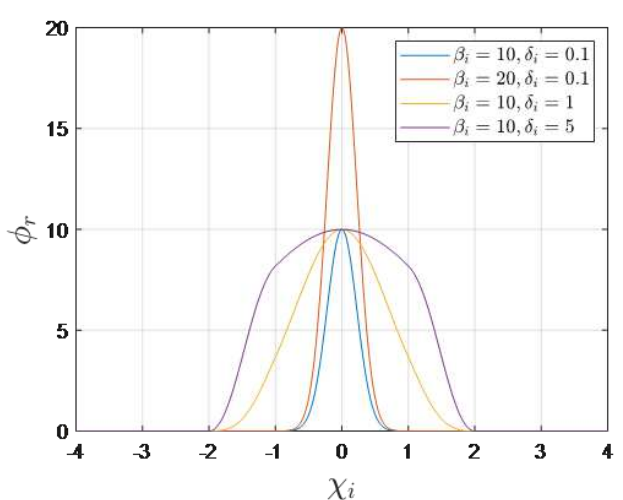

Fig. 3 The influence of parameters on the obstacle repulsion field.

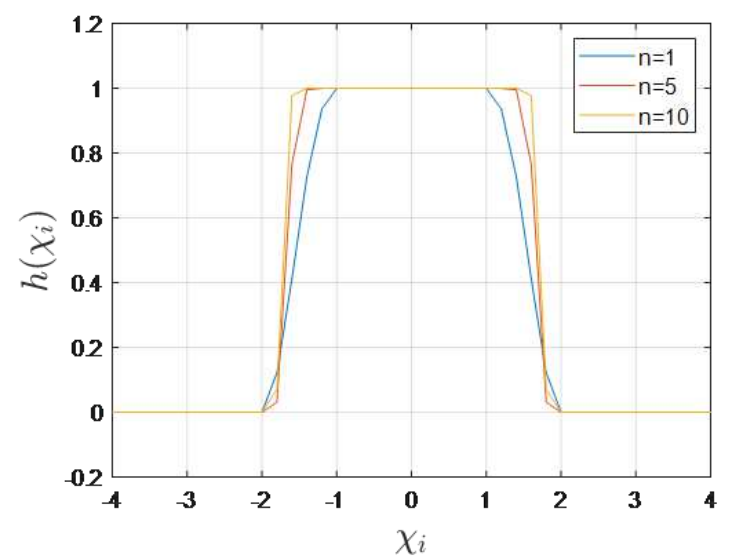

Fig. 4 n-th order smooth switching function $h(\chi)\left(\chi_{a}=\right.$ $\left.2, \chi_{b}=1\right)$.

To illustrate the effect of the smoothness of the n-th order switching function, the first and second derivatives of $h\left(\chi_{i}\right)$ with $\chi_{i}$ are

$$
\begin{aligned}
& \frac{d h}{d \chi_{i}}= \begin{cases}\lambda, \chi_{b} \leq\left|\chi_{i}\right| \leq \chi_{a}, \\
0, & \text { overwise },\end{cases} \\
& \frac{d^{2} h}{d \chi_{i}{ }^{2}}=\left\{\begin{array}{cc}
-\left(\Theta_{1}+\Theta_{2}+\Theta_{3}\right), \chi_{b} \leq\left|\chi_{i}\right| \leq \chi_{a}, \\
0, & \text { overwise, }
\end{array}\right.
\end{aligned}
$$

where $\rho=\frac{\pi \chi_{i}^{2}}{\chi_{b}^{2}-\chi_{a}^{2}}, \varpi=\frac{\pi}{2} \frac{\chi_{i}^{2}-\chi_{b}^{2}}{\chi_{a}^{2}-\chi_{b}^{2}}, \varsigma=\frac{\pi}{2} \sin ^{n}(\varpi), \lambda=$ $-\frac{\pi^{2}}{2} n^{2} \cos ^{n-1}(\varsigma) \sin (\varsigma) \sin ^{n-1}(\varpi) \cos (\varpi) \frac{\chi_{i}}{\chi_{a}^{2}-\chi_{b}^{2}}, \Theta_{1}=(n$ -1) $\rho \cos ^{2}(\varpi)\left[\cos (\varsigma) \sin (\varsigma)-\sin ^{2}(\varsigma) \sin ^{n}(\varpi)\right], \Theta_{2}=\sin (\varpi)$ $\cos (\varsigma) \sin (\varsigma)[\cos (\varpi)-(n-1) \sin (\varpi) \rho], \Theta_{3}=\frac{n \pi}{2} \cos ^{2}(\varsigma)$ $\sin ^{n}(\varpi) \cos ^{2}(\varpi) \rho$. Since $\frac{d^{2} h}{d \chi^{2}}$ has a larger value when the order is larger, this paper uses $\ln (a)$ to represent the relationship between $\frac{d^{2} h}{d \chi_{i}{ }^{2}}$ and $\chi$, where $a=\left|\frac{d^{2} h}{d \chi_{i}{ }^{2}}+1\right|$. It can be seen that $h\left(\chi_{i}\right)$ is second-order continuous divergence, which can meet the design requirements of the robot controller. From Fig. 5(a)-5(b), it can be found that as the order increases, the change rate of 


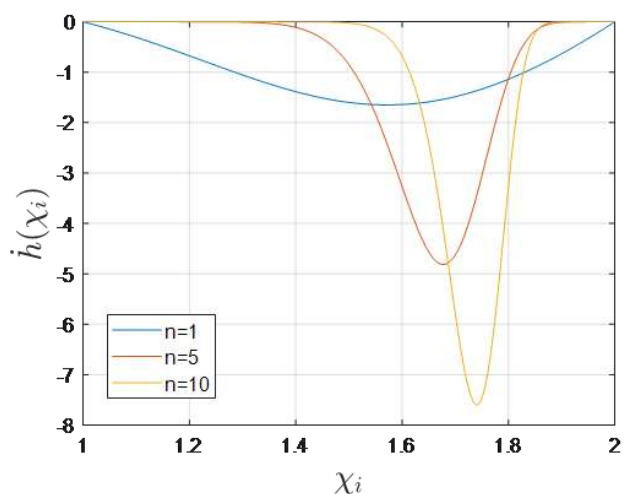

(a)

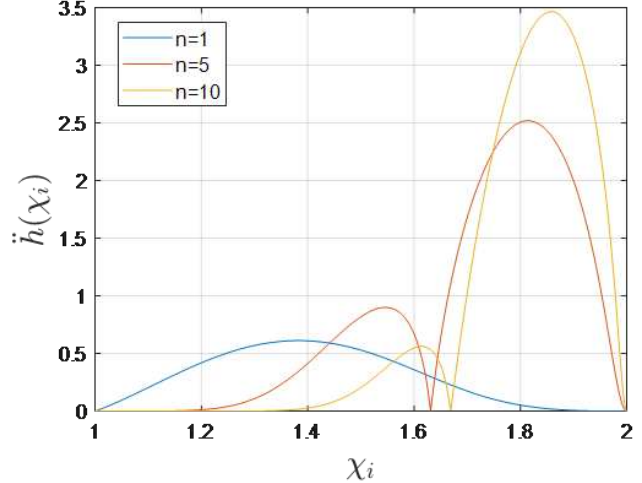

(b)

Fig. 5 The first derivative (a), The second derivative (b).

the switching node $\frac{d h}{d \chi_{i}}$ at $(1,0)$ becomes smaller, so the smoothness at this node becomes stronger.

\subsubsection{Barrier Lyapunov Function design}

The traditional Barrier Lyapunov Function sets the value of the function for the restricted region of the desired state to infinity, the value of the function for the desired state to a minimum (usually 0), and designs the controller so that the function decreases with time. By combining (6) and (20), we design the following Gaussian Barrier Lyapunov Function for the existence of multiple obstacles,

$V=\frac{x^{2}+y^{2}}{2}+\sum_{i=1}^{N} \beta_{i} e^{-\frac{\chi_{i}^{2}}{\delta_{i}}} h\left(\chi_{i}\right)$.

Remark 3 For the convenience of reading, define the target point in this section as $(0,0)$ and the robot position coordinates as $(x, y)$. Moreover, the Gaussian Barrier Lyapunov Function $V$ designed in this paper is different from the traditional Barrier Lyapunov function, and when the parameters are close to the bounded limit, the Gaussian Barrier Lyapunov Function does not increase to infinity in the restricted state region.

\subsubsection{Anti-saturation obstacle avoidance controller}

To achieve the purpose of obstacle avoidance, the following controller is designed,

$u=-\tanh \left[K_{1} \sqrt{x_{s}^{2}+y_{s}^{2}} p\left(\psi_{s}-\psi\right)\right] u_{\max }$,

$\omega=\frac{\dot{y}_{s} x_{s}-y_{s} \dot{x}_{s}}{x_{s}^{2}+y_{s}^{2}}+K_{s} \tanh \left(\psi_{s}-\psi\right)$,

where

$x_{s}=x+\sum_{i=1}^{N} \beta_{i} e^{-\sigma_{i}}\left(-\frac{2 h}{\delta_{i}}-\Delta s\right)\left(x-x_{i}\right)$

$y_{s}=y+\sum_{i=1}^{N} \beta_{i} e^{-\sigma_{i}}\left(-\frac{2 h}{\delta_{i}}-\Delta s\right)\left(y-y_{i}\right)$, $\psi_{s}=\operatorname{atan} 2\left(y_{s}, x_{s}\right)$,

where the parameters $K_{1}$ and $K_{s}$ meet the following constraints,

$K_{1} u_{\max } \leq \omega_{\max }, \quad K_{s}>0$.

\subsubsection{Stability analysis}

Theorem 2 Considering the wheeled robot dynamic system (1), the $n$-th order smooth switching function (19) and the Gaussian Barrier Lyapunov Function (21), and designing the control law (22) based on the constraints (25), then the wheeled robot is globally asymptotically stable without obstacle collision.

Proof. The time derivative of equation (21) is

$$
\begin{aligned}
\dot{V}= & x u \cos \psi+y u \sin \psi+\sum_{i=1}^{N} \beta_{i} e^{-\sigma_{i}} \\
& \left\{-\frac{2 h}{\delta_{i}}\left[\left(x-x_{i}\right) u \cos \psi+\left(y-y_{i}\right) u \sin \psi\right]+\dot{h},\right\}
\end{aligned}
$$

where $\sigma_{i}=\frac{\left(x-x_{i}\right)^{2}+\left(y-y_{i}\right)^{2}}{\delta_{i}}$, To facilitate calculation, let $\dot{h}=\frac{d h}{d \chi_{i}} \dot{\chi}_{i}=-\Delta_{s}\left[\left(x-x_{i}\right) u \cos \psi+\left(y-y_{i}\right) u \sin \psi\right]$

where $\Delta_{s}=\frac{d h}{d \chi_{i}} \frac{1}{\chi_{i}} \cdot \dot{h}$ is the derivative of $h\left(\chi_{i}\right)$ with respect to the robot position $(x, y)$. Therefore, the above two equations can be combined to obtain

$$
\begin{gathered}
\dot{V}=u \cos \psi\left[\sum_{i=1}^{N} \beta_{i} e^{-\sigma_{i}}\left(-\frac{2 h}{\delta_{i}}-\Delta_{s}\right)\left(x-x_{i}\right)+x\right]+ \\
u \sin \psi\left[\sum_{i=1}^{N} \beta_{i} e^{-\sigma_{i}}\left(-\frac{2 h}{\delta_{i}}-\Delta_{s}\right)\left(y-y_{i}\right)+y\right] .
\end{gathered}
$$

From equation (23), the above equation can be simplified as $\dot{V}=u x_{s} \cos \psi+u y_{s} \sin \psi$, and $x_{s}$ represents the 
combined force of attraction and repulsion in the $x$-axis direction of the robot, and similarly $y_{s}$ represents the combined force of attraction and repulsion in the $y$-axis direction. Then the above equation can be written as

$\dot{V}=u \sqrt{x_{s}^{2}+y_{s}^{2}} \cos \left(e_{\psi_{s}}\right)$.

Define the angle error $e_{\psi_{s}}$ as

$e_{\psi_{s}}=\psi_{s}-\psi$

where $e_{\psi_{s}}$ has a different meaning from $e_{\psi}$ in the equation (4). According to the definition of $h\left(\chi_{i}\right)$ and $\psi_{s}$, when $\left|\chi_{i}\right|>\chi_{a}, \psi_{s}=\operatorname{atan} 2(x, y)$, at this time $\psi_{s}$ has the same meaning as $\alpha$ in (3), so when the robot is outside the repulsive field, $e_{\psi_{s}}$ is the same as $e_{\psi}$. When the robot is in the range of the repulsive field, $\psi_{s}$ is the desired heading angle of the robot, and $\psi$ is the actual heading angle of the robot. Therefore, $e_{\psi_{s}}$ must be controlled in order to enable the robot to avoid obstacles.

According to the definition of $e_{\psi_{s}}, x_{s}, y_{s}, \psi_{s}$, we get $\dot{e}_{\psi_{s}}=\frac{\dot{y}_{s} x_{s}-y_{s} \dot{x}_{s}}{x_{s}^{2}+y_{s}^{2}}-\omega$. where

$$
\begin{aligned}
\dot{y}_{s} x_{s}-y_{s} \dot{x}_{s} & =\sqrt{x_{s}^{2}+y_{s}^{2}}\left(\dot{y}_{s} \frac{x_{s}}{\sqrt{x_{s}^{2}+y_{s}^{2}}}-\dot{x}_{s} \frac{y_{s}}{\sqrt{x_{s}^{2}+y_{s}^{2}}}\right) \\
& =u \sqrt{x_{s}^{2}+y_{s}^{2}}\left(y_{s 1} \cos \psi_{s}-x_{s 1} \sin \psi_{s}\right) .
\end{aligned}
$$

Combine the above two equations to get

$$
\begin{aligned}
\dot{e}_{\psi_{s}} & =\frac{u \sqrt{x_{s}^{2}+y_{s}^{2}}\left(y_{s 1} \cos \psi_{s}-x_{s 1} \sin \psi_{s}\right)}{x_{s}^{2}+y_{s}^{2}}-\omega \\
& =\frac{u\left(y_{s 1} \cos \psi_{s}-x_{s 1} \sin \psi_{s}\right)}{d_{s}}-\omega .
\end{aligned}
$$

Among them, $\dot{x}_{s}=u x_{s 1}, \dot{y}_{s}=u y_{s 1}, d_{s}=\sqrt{x_{s}^{2}+y_{s}^{2}}$. Because of the boundedness of $e_{\psi_{s}}, x_{s 1}$ and $y_{s 1}$ can be expressed as $x_{s 1}=\cos \psi+\sum_{i=1}^{N} \beta_{i} e^{-\sigma_{i}}\left[\left\{\frac{4 \varrho}{\delta_{i}}\left(\frac{h}{\delta_{i}}+\right.\right.\right.$ $\left.\left.\left.\Delta_{s}\right)-\frac{\Delta_{\dot{s}}}{u}\right\}\left(x-x_{i}\right)-\left(\frac{2 h}{\delta_{i}}+\Delta_{s}\right) \cos \psi\right], y_{s 1}=\sin \psi+$ $\left.\sum_{i=1}^{N} \beta_{i} e^{-\sigma_{i}}\left[\left\{\frac{4 \varrho}{(} \frac{h}{\delta_{i}}+\Delta_{s}\right)-\frac{\Delta_{\dot{s}}}{u}\right\}\left(y-y_{i}\right)-\left(\frac{2 h}{\delta_{i}}+\Delta_{s}\right) \sin \psi\right]$. where $\Delta_{\dot{s}}=-\frac{\pi^{2}}{2} n^{2}\left[A \frac{\pi^{2}}{2} n(n-1) \cos ^{n-2}(\varsigma) \sin ^{n-1}(\varpi) \gamma+\right.$ $B \frac{\pi^{2}}{2} n \cos (\varsigma) \sin ^{n-1}(\varpi) \gamma+C \pi(n-1) \sin ^{n-2}(\varpi) \gamma-D \pi$ $\sin (\varpi) \gamma] \dot{\chi}_{i}-\dot{h} \frac{1}{\chi_{i}^{2}}, \varrho=\left(x-x_{i}\right) \cos \psi+\left(y-y_{i}\right) \sin \psi$. And $\gamma=\frac{1}{\chi_{a}^{2}-\chi_{b}^{2}}, A=\sin (\varsigma) \sin ^{n-1}(\varpi) \cos (\varpi) \gamma, B=$ $\cos ^{n-1}(\varsigma) \sin ^{n-1}(\varpi) \cos (\varpi) \gamma, \quad C=\cos ^{n-1}(\varsigma) \sin (\varsigma)$ $\cos (\varpi) \gamma, D=\cos ^{n-1}(\varsigma) \sin (\varsigma) \sin ^{n-1}(\varpi) \gamma$, and $\dot{\chi}_{i}=$ $u \frac{\varrho}{\chi_{i}}$.

The equation (29) is different from (5). $d$ only represents the distance between the robot and the target point. The positioning controller controls $d \rightarrow 0$ in order to make the robot reach the target point and keep it stable. But in the obstacle avoidance control, it is necessary to control not only the distance $d$ but also trajectory of the robot when it moves in the repulsive force field. When $\left|\chi_{i}\right|>\chi_{a}$, the robot is not in the repulsive force field, and $d_{s}=\sqrt{x^{2}+y^{2}}=d$. When the robot enters the repulsive field, $h\left(\chi_{i}\right)$ goes from 0 to 1 and the repulsive force field starts to work. At this time, $d_{s}=\sqrt{x_{s}^{2}+y_{s}^{2}}$, so $d_{s}$ varies with $\chi$. Then equation (29) can be expressed as

$$
\begin{aligned}
\dot{e}_{\psi_{s}} & =\frac{u \sqrt{x_{s}^{2}+y_{s}^{2}}\left(y_{s 1} \cos \psi_{s}-x_{s 1} \sin \psi_{s}\right)}{x_{s}^{2}+y_{s}^{2}}-\omega \\
& =-K_{s} \tanh \left(e_{\psi_{s}}\right) .
\end{aligned}
$$

From (28) and (30), the signs of $e_{\psi_{s}}$ and $\dot{e}_{\psi_{s}}$ are always opposite, so $e_{\psi_{s}} \rightarrow 0$.

In summary, substituting the controller (22) into (27), then we will get

$$
\begin{aligned}
\dot{V} & =u \sqrt{x_{s}^{2}+y_{s}^{2}} \cos \left(e_{\psi_{s}}\right)=u d_{s} \cos e_{\psi_{s}} \\
& =-\tanh \left[K_{1} d_{s} p\left(e_{\psi_{s}}\right)\right] d_{s} u_{\max } \cos \left(e_{\psi_{s}}\right) \\
& \leq 0 .
\end{aligned}
$$

According to Lemma 1, we can get: $x_{s} \rightarrow 0, y_{s} \rightarrow 0$ as $t \rightarrow \infty$. Therefore, according to the definition of $x_{s}, y_{s}$, $h\left(\chi_{i}\right)$, if $\chi_{i}>\chi_{a}$ when the robot stops, then at $t \rightarrow \infty$, $x \rightarrow 0, y \rightarrow 0$, that is, the robot is stable at the target point. The proof is completed.

Remark 4 In this section, a smooth artificial potential field repulsive field function is constructed by introducing Gaussian function and the smooth switching function, which solves the edge chattering problem of traditional repulsive field function. The Gaussian Barrier Lyapunov Function is designed to constrain the linear and angular velocities of the robot so that the robot can simultaneously avoid the obstacle and stabilize at the target point.

\section{Simulation results}

\subsection{Simulation of the positioning problem}

Fig. 6 represents the simulation results of the control method (9). Among them, control law parameter are selected as: $K_{1}=4, K_{p}=0.75, K_{i}=0.00007, u_{\max }=$ 0.05 . To verify that the robot can reach the target point from different starting points under the control law (9), we choose the following five starting points: $(-0.4,0)$, $(0.4,0.4),(-0.8,-0.8),(0.1,-0.4),(-0.4,0.5)$. To facilitate observation, select the target point $\left(x_{p}, y_{p}\right)$ as $(0,0)$. The simulation results show that at any starting point, the robot with control law designed in (9) can reach the target point.

In order to verify the advantages of the control law (9) designed in this paper, it is simulated and compared with the control law of the literature [17], as shown 


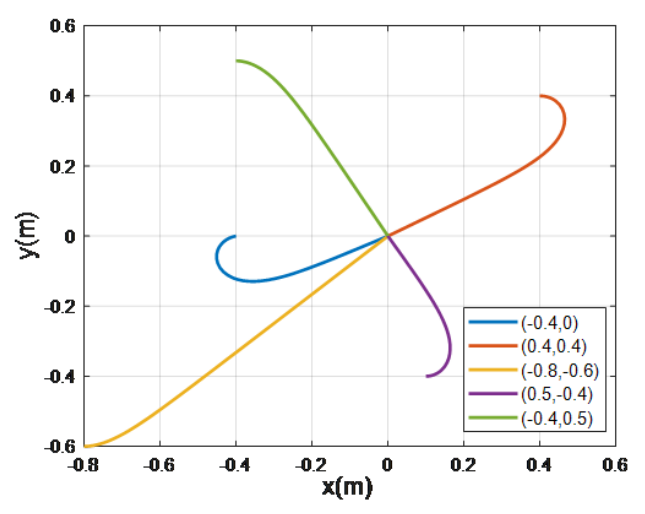

Fig. 6 Position control from different starting points.

in Fig.7-Fig. 8. Because the restriction condition (11) of the control law in this paper is different from that of the literature [17], the selection of $K_{1}$ parameter in this paper is also different from the literature [17]. The parameters of the control law in this paper are selected as follows: $K_{1}=4, K_{p}=0.75, K_{i}=0.00007, u_{\max }=$ 0.05. In [17], the parameters selected for the control law simulation experiment are as follows: $K_{1}=0.1, K_{p}=$ $0.75, K_{i}=0.00007, u_{\max }=0.05$. Select the starting point $T_{p}(-0.4,0)$ and the target point $T_{d}(0.8,0)$.

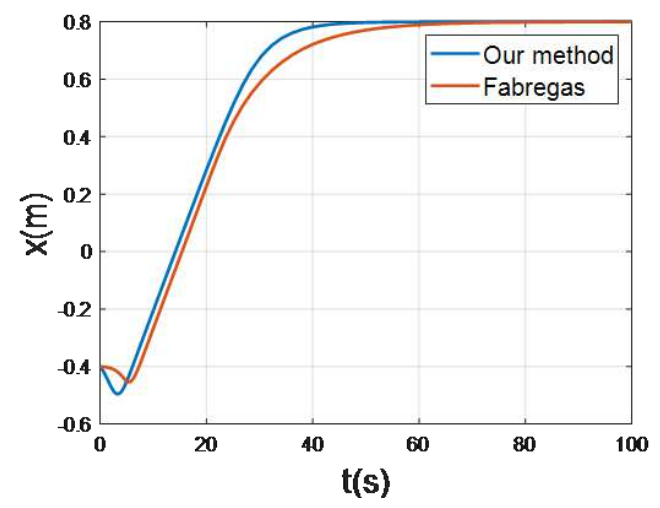

Fig. 7 Horizontal position comparison.

Fig. 7 shows the comparison of the lateral position variation of the robot with different control laws. It can be seen from Fig. 8 that the control law (9) reaches the target point about 10 seconds earlier than the literature [17]. Figure 8 shows the trajectory of the robot linear velocity for different control laws. Under the control law (9) the robot reaches $u_{\max }$ faster. Since the control law in this paper is a continuous control law, the trajectory of the control law (9) in this paper is smoother compared to that in the literature [17], and thus avoids the robot chattering problem.

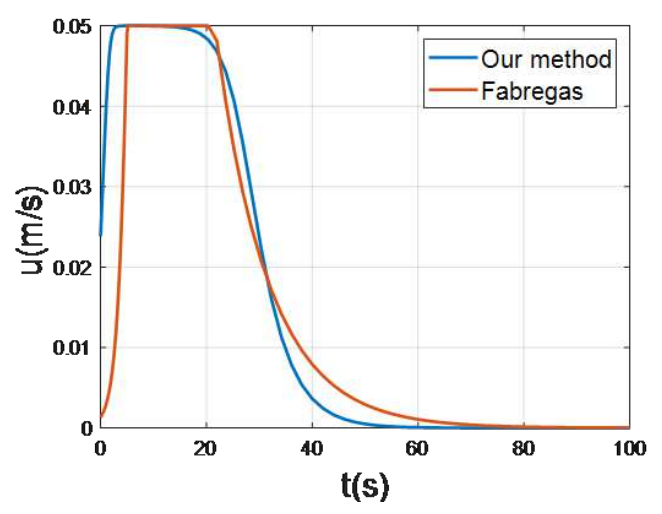

Fig. 8 Linear velocity comparison.

Table 1 Algorithm Parameters.

\begin{tabular}{|l|l|}
\hline $\begin{array}{l}\text { Simulation param- } \\
\text { eters }\end{array}$ & value \\
\hline$\beta_{i}$ & 100 \\
$\delta_{i}$ & 1 \\
$n$ & 1 \\
$K_{1}$ & 0.1 \\
$u_{\max }$ & 0.2 \\
$K_{s}$ & 1 \\
$\chi_{a}$ & 2 \\
$\chi_{b}$ & 1 \\
\hline
\end{tabular}

4.2 Simulation of obstacle avoidance problem

The simulation results of the artificial potential field method of obstacle avoidance for the control law (22)(25) is shown in Fig.9-Fig.13. Let the robot starting point $T_{p}(10,10)$ and target point $T_{d}(0,0)$.

For the convenience of observation, the simulation of avoiding single obstacles and the simulation of avoiding double obstacles adopt the same algorithm parameters, as shown in Table 1.

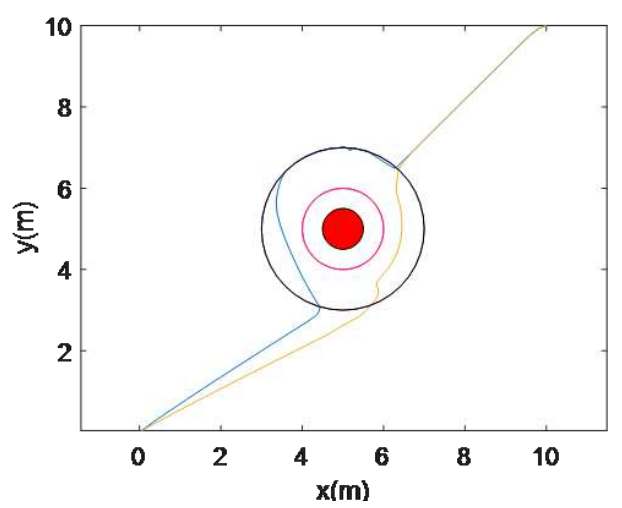

Fig. 9 The difference between $h\left(\chi_{i}\right)$ and $g$ in the case of obstacle avoidance. 
Fig.10 shows the comparison of obstacle avoidance effect between control laws (18) and (20). The black circle is the maximum influence range $\chi_{a}$ of the obstacle repulsion field, and the purple circle is the minimum safety distance $\chi_{b}$ of the obstacle. The yellow line is the simulation trajectory of Equation (20) using the smooth switching function $h\left(\chi_{i}\right)$, and The blue line is the simulated trajectory of equation (18). After entering the repulsive field, the robot's route changes. As can be seen from the blue line the robot may wobble due to the large repulsive force immediately upon entry and the large reaction right at the edge of the repulsive field. And the yellow line can be seen from the robot into the range after the repulsive force field, the repulsive force gradually increased, so the robot will slowly avoid the obstacles. Obviously, the robot trajectory of (20) control law is shorter and smoother.

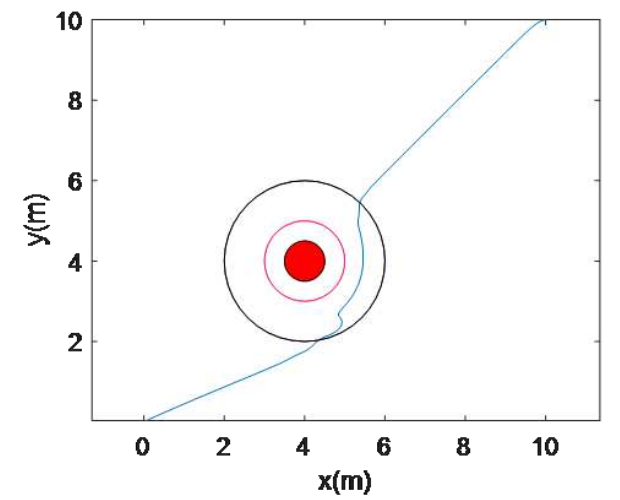

Fig. 10 The trajectory of the robot at obstacles $(4,4)$.

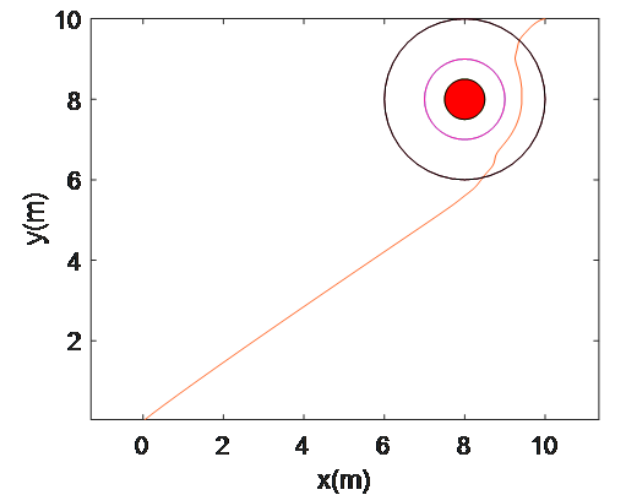

Fig. 11 The trajectory of the robot at obstacles $(8,8)$.

Fig.10-Fig.11 shows the path of the robot when the robot moves from $T_{p}(10,10)$ to $T_{d}(0,0)$ under the control law in this paper. The blue line is the trajectory of the robot when the obstacle is at $(4,4)$. The orange line is the trajectory of the robot when the obstacle is at $(8,8)$, indicating that when the robot moves, no matter where there is an obstacle or obstacle repulsion field in the path of the robot, the robot can make obstacle avoidance responses.

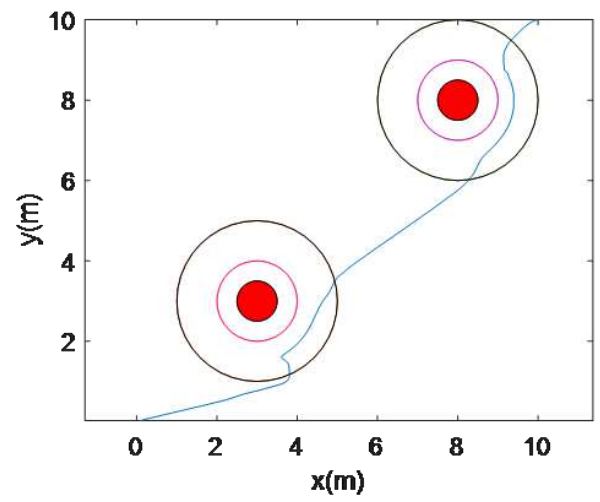

Fig. 12 Position control in case of double obstacles.

The blue line in Fig.12 is the robot motion path, which is a simulation diagram of the position control of the robot continuously avoiding two obstacles. The design has obstacles at the coordinates $(3,3)$ and $(8,8)$, and the robot avoids the second obstacle in the same mode after avoiding the first obstacle.

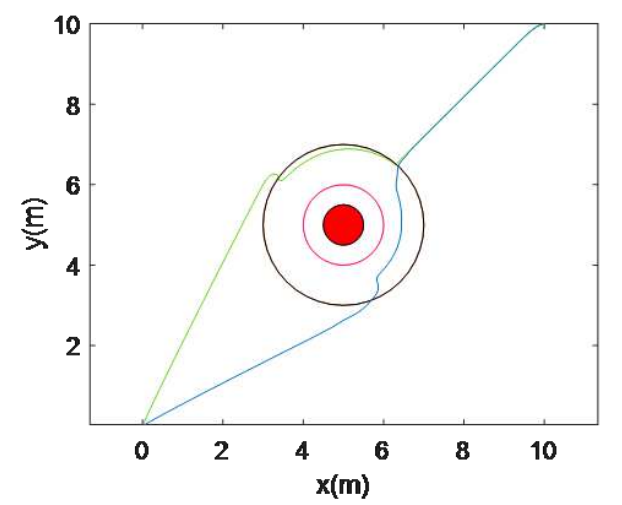

Fig. 13 Simulation comparison of different obstacle avoidance algorithms.

Fig. 13 shows the comparison of the simulation routes between the traditional obstacle avoidance algorithm and the proposed obstacle avoidance algorithm in this paper. The blue line is the simulation route of the proposed algorithm, and the green line is the simulation route of the traditional obstacle avoidance algorithm. It can be found that the traditional obstacle avoidance algorithm is more conservative than the algorithm proposed in this paper, and it starts to move away from 
the obstacle when it is far away from the obstacle. Compared with the traditional algorithm, the trajectory of the robot is shorter in this paper.

\section{Conclusion}

This paper proposes a novel control law that drives the robot to the desired point and achieves the obstacle avoidance. The linear velocity control law that can effectively overcome the saturation problem is used to improve the existing positioning control law. The Gaussian function and the smooth switching function are introduced to improve the artificial potential field, and the Gaussian Barrier Lyapunov Function based on the artificial potential field method is designed, so that the robot can avoid one or more obstacles while achieving the desired target point. The control law designed in this paper is continuous and smooth, and can effectively avoid obstacles. Simulation results show that the control laws proposed in this paper compared with the existing literature have a significant improvement.

The obstacle avoidance control law is designed based on the artificial potential field method. Although it solves the problem of chattering phenomenon at the edge of the repulsion field, it does not solve the problem of the unreachable target point and local minimum of the artificial potential field. In the follow-up research, it will be considered in combination with the optimization algorithms.

Acknowledgments This paper was funded by the general projects of science and technology under grant 2021 XJKJ04, a project supported by scientific research fund of Zhejiang provincial education department under grant Y202146005.

Declarations The authors have no relevant financial or non-financial interests to disclose. All authors contributed to the study conception and design, and all authors commented on previous and final versions of the manuscript. All data generated or analysed during this study are included in this published article (and its supplementary information files).

\section{References}

1. J. L. Mata-Machuca, L. F. Zarazua and R. Aguilar-Lpez, "Experimental Verification of the Leader-Follower Formation Control of Two Wheeled Mobile Robots with Obstacle Avoidance," IEEE Latin America Trans., vol. 19, no. 8, pp. 1417-1424, Aug. 2021.

2. J. Liao, Z. Chen and B. Yao, "Model-Based Coordinated Control of Four-Wheel Independently Driven Skid Steer Mobile Robot with WheelGround Interaction and Wheel
Dynamics," IEEE Trans. Industr. Inform., vol. 15, no. 3, pp. 1742-1752, March 2019.

3. J. V. Salazar Luces, S. Matsuzaki and Y. Hirata, "RoVaLL: Design and Development of a Multi-Terrain Towed Robot With Variable Lug-Length Wheels," IEEE Robot Autom. Lett., vol. 5, no. 4, pp. 6017-6024, Oct. 2020.

4. A. M. Bloch, M. Reyhanoglu, and N. H. McClamroch, "Control and stabilization of nonholonomic dynamic systems," IEEE Trans. Autom. Control, vol. 37, no. 11, pp. 1746-1757, Nov. 1992.

5. C. C. de Wit and O. Sordalen, "Exponential stabilization of mobile robots with nonholonomic constraints," IEEE Trans. Autom. Control, vol. 37, no. 11, pp. 1791-1797, Nov. 1992.

6. A. Widyotriatmo and K. S. Hong, "Navigation functionbased control of multiple wheeled vehicles," IEEE Trans. Ind. Electron, vol. 58, no. 5, pp. 1896-1906, Apr. 2011.

7. X. Yu and L. Liu, "Distributed formation control of nonholonomic vehicles subject to velocity constraints," IEEE Trans. Ind. Electron., vol. 63, no. 2, pp. 1289-1298, Nov. 2016.

8. G. Caprari, T. Estier , and R. Siegwart, "Fascination of Down Scaling - Alice the Sugar Cube Robot," Journal of Micromechatronics, vol. 1, no. 3, pp. 177-189, Jul. 2014.

9. S. K. Malu and J. Majumdar, "Kinematics, localization and control of differential drive mobile robot," Glob. J. Res. Eng., vol. 14, no. 1, 2014.

10. F. Pourboghrat and M. P. Karlsson, "Adaptive control of dynamic mobile robots with nonholonomic constraints," Comput. Electr. Eng., vol. 28, no. 4, pp. 241 - 253, Jul. 2002.

11. Y. Ma, V. Cocquempot, M. E. B. El Najjar, and B. Jiang, "Actuator failure compensation for two linked $2 \mathrm{wd}$ mobile robots based on multiple-model control," Int. J. Appl. Math. Comput. Sci., vol. 27, no. 4, pp. 763-776, Dec. 2017.

12. J. Wang, M. Q. H. Meng and O. Khatib, "EB-RRT: Optimal Motion Planning for Mobile Robots," IEEE Trans. Autom. Sci. Eng., vol. 17, no. 4, pp. 2063-2073, Oct. 2020

13. J. S. Zelek, M. D. Levine, "Local-global concurrent path planning and execution," IEEE Trans. Syst Man Cybern C Appl Rev., vol. 30, no. 6, pp. 865-870, Nov. 2000.

14. P. Shi, Y. Zhao, "Global path planning for mobile robot based on improved artificial potential function," IEEE ICAL, Shenyang, On, CHN, 2009, pp. 1900-1904.

15. Y. Koren and J. Borenstein, "Potential fifield methods and their inherent limitations for mobile robot navigation," IEEE Int. Conf. Robot. Autom., Washington, DC, USA, May 11-15, 2002.

16. V. J. G. Villela, R. Parkin, M. L. Parra, "A wheeled mobile robot with obstacle avoidance capability," Tecnologa Y Desarrollo, vol. 1, no. 5, pp. 159-166, Sep. 2004.

17. E. Fabregas, G. Farias, E. Aranda-Escolstico, "Simulation and Experimental Results of a New Control Strategy For Point Stabilization of Nonholonomic Mobile Robots," IEEE Trans. Ind. Electron., vol. 67, no. 8, pp. 6679-6687, Aug. 2020.

18. D. Galan, E. Fabregas, G. Garcia, J. Saenz, G. Farias, S. Dormido-Canto, and S. Dormido, "Online virtual control laboratory of mobile robots," IFAC-PapersOnLine, vol. 51, no. 4, pp. 316-321, Jun. 2018.

19. M. Mancini, N. Bloise, E. Capello. and E. Punta, "Sliding mode control techniques and artifificial potential fifield for dynamic collision avoidance in rendezvous maneuvers, IEEE Control Syst Lett., vol. 4, no. 2, pp.313-318, Jul. 2020.

20. J. Muoz and N. Fitz-Coy, "Rapid path-planning option$\mathrm{s}$ for autonomous proximity operations of spacecraft," in 
Proc. AIAA/AAS Astrodyn. Spec. Conf., Toronto, ON, Canada, 2010, pp. 1-24.

21. Richard, Zappulla, Hyeongjun, "Real-Time Autonomous Spacecraft Proximity Maneuvers and Docking Using an Adaptive Artificial Potential Field Approach," IEEE Trans. Control Syst. Technol., vol. 27,no. 6, pp. 2598-2605, Sep. 2019.

22. D. B. Payne and J. R. Stern, "Wavelength-switched pas- sively coupled single-mode optical network," in Proc. IOOC-ECOC, Boston, MA, USA, 1985, pp. 585-590.

23. J.Wu, W.Chen, D. Zhao, "Globally stable direct adaptive backstepping NN control for uncertain nonlinear strictfeedback systems," Neurocomputing, vol. 122, pp.134-147, Dec. 2013. 